\title{
Prevalencia del síndrome de Burnout en los residentes de cirugía general de Chile y sus factores asociados
}

\author{
Nelson Muñoz P. ${ }^{1}$, Nicolás Campaña W. ${ }^{1}$ y Gonzalo Campaña V. ${ }^{1,2}$
}

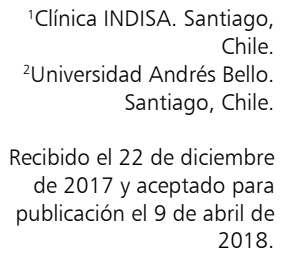

Correspondencia a:

Dr. Gonzalo Campaña V. gonzalo.campana@indisa.cl
Prevalence of Burnout syndrome in general surgery residents of Chile and its associated factors

Introduction: The Burnout Syndrome (SBO), corresponds to a response to chronic work stress conformed by negative attitudes and feelings. It is characterized by the presence of emotional exhaustion, depersonalization and low personal fulfillment.Objective: To evaluate the presence of SBO in residents of general surgery in Chile. Patients and Method: Cross-sectional study, with an invitation to answer an anonymous online survey. The instrument used was the Maslach Burnout Inventory of 22 questions, which is divided into 3 subscales, emotional exhaustion (AE), depersonalization (D) and low personal fulfillment (RP). These in turn are divided into low, moderate or high. SBO is defined by high grade in AE or D, or low grade of RP. Results: 103 complete answers were obtained, of which $72.8 \%$ met SBO criteria on at least one subscale. In the multivariate logistic regression model, age appears as a protective factor with an OR 0.8 (95\% CI 0.64-0.99) and having children as a risk factor with an OR 4.94 (95\% CI 1.03-23.52). In the AE subscale, women presented a higher risk of developing SBO RRR 5.32 (95\% CI 1.10-25.66), while in the RP subscale, there was an increased risk of developing SBO in those who performed the specialty in metropolitan region RRR 5.43 (95\% CI 1.12-26.37). Conclusions: The prevalence of SBO in residents of general surgery in Chile is $72.8 \%$, mainly in young people, with children, women and the metropolitan region.

Key words: professional burnout; general surgery; medical residency.

\section{Resumen}

Introducción: El Síndrome de Burnout (SBO), corresponde a una respuesta al estrés laboral crónico conformado por actitudes y sentimientos negativos. Se caracteriza por la presencia de agotamiento emocional, despersonalización y baja realización personal. Objetivo: Evaluar la presencia del SBO en residentes de cirugía general en Chile. Pacientes y Método: Estudio de corte transversal, con invitación a responder encuesta anónima online. El instrumento utilizado, fue el Maslach Burnout Inventory de 22 preguntas, que se divide en 3 subescalas, agotamiento emocional (AE), despersonalización (D) y baja realización personal (RP). Éstas a su vez se dividen en grado bajo, moderado o alto. SBO se define por grado alto en AE o D, o grado bajo de RP. Resultados: Se obtuvieron 103 respuestas completas, de las cuales $72,8 \%$ cumple criterios de SBO en al menos una subescala. En el modelo de regresión logística multivariado, la edad aparece como un factor protector con un OR 0,8 (IC 95\% 0,64-0,99) y el tener hijos como un factor de riesgo con un OR 4,94 (IC 95\% 1,03-23,52). En la subescala de AE, las mujeres presentan mayor riesgo de desarrollar SBO RRR 5,32 (IC 95\% 1,10-25,66), mientras que en la subescala de RP, hubo un mayor riesgo de desarrollar SBO en quienes realizan la especialidad en Región Metropolitana RRR 5,43 (IC 95\% 1,12-26,37). Conclusiones: La prevalencia del SBO en los residentes de cirugía general de Chile es de un 72,8\%, principalmente en jóvenes, con hijos, mujeres y de región metropolitana.

Palabras clave: burnout profesional; cirugía general; médico residente. 


\section{Introducción}

El Síndrome de Burnout (SBO), corresponde a una respuesta al estrés laboral crónico conformado por actitudes y sentimientos negativos hacia las personas con las que se trabaja y hacia el propio rol profesional. Se caracteriza por la presencia de agotamiento emocional, despersonalización y baja realización personal. El agotamiento emocional es el elemento clave del síndrome, refiriéndose a que los sujetos se presentan emocionalmente agotados y sobrepasados en cuanto a sus recursos emocionales. La despersonalización o cinismo, involucra actitudes negativas, generando sentimientos distantes hacia otras personas. La baja realización personal se refiere a la disminución de los sentimientos de competencia y éxito, y a autoevaluarse negativamente, especialmente en lo laboral ${ }^{1}$.

Los problemas derivados del estrés laboral crónico provocan accidentabilidad, ausentismo y baja productividad $^{2,3}$, teniendo repercusión directa en el desempeño, calidad y seguridad en la atención de pacientes ${ }^{4}$.

El SBO no sólo se presenta en los médicos en formación, sino que en todo el personal de salud. Así lo revelan estudios nacionales ${ }^{5-7}$. Referencias internacionales recientemente publicadas han descrito que más del $50 \%$ de los médicos han presentado SBO a lo largo de su carrera, siendo en gran parte durante el período de formación de médico o residente ${ }^{8,9}$. Específicamente en residentes de cirugía general la encuesta nacional norteamericana del año 2016, reveló una prevalencia de un $69 \%$ de $\mathrm{SBO}^{10}$.

En Chile no existen estudios en residentes de cirugía general y es por esto que nos planteamos como objetivo principal determinar la prevalencia del SBO en ellos mediante la aplicación del Maslach Burnout Inventory - Human Service Survey (MBIHSS) y como objetivos secundarios establecer las características sociodemográficas asociadas.

\section{Materiales y Método}

El diseño es un corte transversal (estudio de prevalencia), cuyo universo corresponde a todos los residentes de cirugía general de Chile. Consideramos como criterios de inclusión el estar cursando programa de formación en Cirugía General, responder encuesta voluntariamente durante el período julio de 2017 y, como criterio de exclusión, el haber respondido de forma incompleta. Se realizó un cálculo del tamaño muestral, mediante el empleo de una estimación de proporción considerando un número total de residentes en 252 , con una confianza del $95 \%$, precisión del $4 \%$ y proporción del $5 \%$, obtuvimos un tamaño muestral necesario de 79 encuestados; considerando una pérdida del $15 \%$, nuestra muestra ajustada a las pérdidas es de 93 . El número total de residentes (252), lo obtuvimos considerando que existen 18 programas de formación, con un promedio de 14 residentes por cada programa. Esta cifra hubo que estimarla, debido a que no hay cifras oficiales al consultar a las sociedades correspondientes.

Definimos como variable dependiente la presencia del SBO. Para poder evaluarlo, el instrumento utilizado fue el Maslach Burnout Inventory - Human Service Survey (MBI-HSS) ${ }^{11}$ cuya validez factorial está aceptada al español ${ }^{12}$ y en profesionales chilenos ${ }^{13,14}$. Este cuestionario consta de 22 ítems evaluados con una escala tipo Likert de 0 a 6 puntos (Tabla 1), está factorizado en 3 subescalas: agotamiento emocional (AE), despersonalización (D) y realización personal (RP), las que representan las tres dimensiones del síndrome. Cada subescala se divide en 3 grados (bajo, moderado y alto), según datos previamente validados. Los valores considerados para cada una de las subescalas son los siguientes, $\mathrm{AE}$ (bajo $\leq 18$, moderado 19-26, alto $\geq 27$ ), D (bajo $\leq 5$, moderado 6-9, alto $\geq 10$ ) y RP (bajo $\geq 34$, moderado 29-33, alto $\leq 28$ ). Se define el SBO, como grado alto en agotamiento emocional o despersonalización o en grado bajo de realización personal. Para la recolección de las encuestas se ocupó la plataforma web SurveyMonkey ${ }^{\circledR}$, con la intención de externalizar las encuestas y garantizar el anonimato del encuestado. En esta misma línea, se destaca también que la encuesta no incluye nombre, RUT, ni fecha de nacimiento.

Otras variables a evaluar fueron la edad, género, estado civil, la presencia de hijos y número de éstos, centro formador, año en curso, financiamiento y horas de jornada laboral.

La estadística utilizada fue descriptiva y analítica. Las variables categóricas se expresaron en números y porcentajes con cálculo de $\chi^{2}$ y las continuas en medias \pm DS, con aplicación de prueba de Mann-Whitney para variables que no distribuyen normal.

Se realizó un modelo de regresión logística multivariable, incluyendo con un $\mathrm{p}<0,05$, con posterior cálculo de Odds Ratio e intervalos de confianza al 95\%. El análisis de los datos se realizó con software STATA 13.0.

Este estudio cuenta con la aprobación del Comité de Bioética de la Universidad Andrés Bello. 
Tabla 1. Cuestionario Burnout de Maslach (MBI)

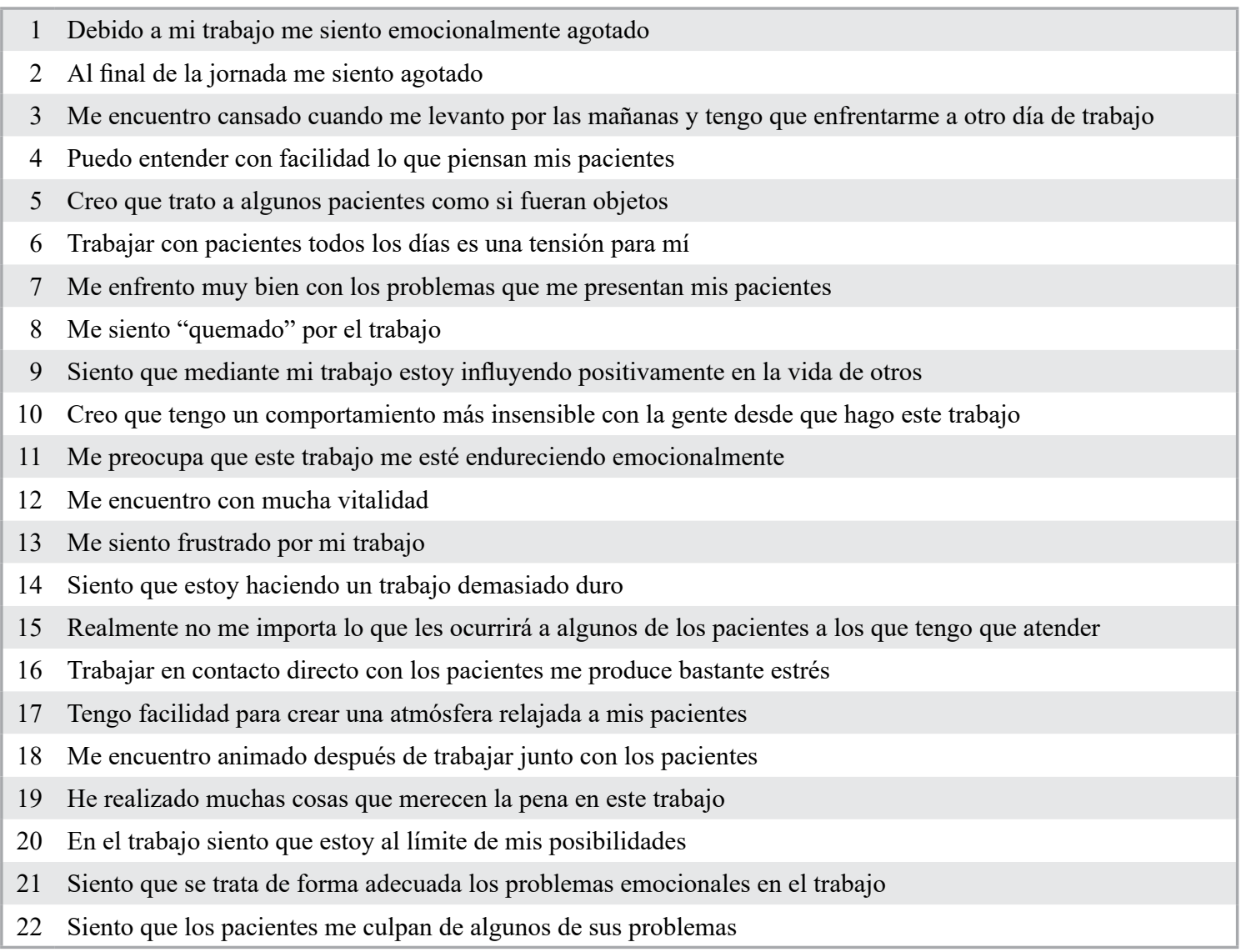

Cada uno de los enunciados se valora en escala Likert de 0 a 6 puntos, correspondiendo $0=$ nunca, $1=$ alguna vez al año o menos, 2 = una vez al mes o menos, 3 = algunas veces al mes, $4=$ una vez a la semana, 5 = varias veces a la semana, $6=$ diariamente

\section{Resultados}

Se obtuvieron un total de 117 respuestas, de las cuales 103 estaban completas (88\%), siendo el género masculino el $67 \%$ de la muestra, con una media de edad de $29,6 \pm 3,0$, el $72 \%$ de los encuestados era soltero o conviviente, el $78 \%$ no tenía hijos y similar cifra realiza su residencia con algún tipo de financiamiento. El perfil biodemográfico completo se expresa en Tabla 2 y la presencia de SBO según centro formador en Tabla 3.

Aplicando el cuestionario MBI, el 72,81\% de los encuestados presenta SBO, de los cuales el $26 \%$, $27 \%$ y $19 \%$ tienen SBO en al menos una, dos y tres subescalas, respectivamente.

En la subescala de agotamiento emocional y despersonalización el $52 \%$ y el $56 \%$ de los encuestados, respectivamente, presentan grado alto, mientras que el $30 \%$ presenta baja realización personal, que son precisamente, los criterios que definen el SBO (Tabla 4).

Al dicotomizar la presencia de SBO y analizar las variables estudiadas mediante un modelo de regresión logística multivariable (Tabla 5), existe una diferencia estadísticamente significativa en la edad $(p=0,042)$ con un OR 0,8 (IC 95\% 0,64-0,99) y el tener hijos $(\mathrm{p}=0,045)$ con un OR 4,94 (IC 95\% $1,03-23,52)$. No hubo diferencias significativas en lo referente al género, estado civil, universidad, año de residencia, financiamiento, ni tampoco al considerar Región Metropolitana vs otras regiones.

Al analizar por subescalas (Tabla 6), vemos que en agotamiento emocional hay una diferencia estadísticamente significativa al analizar el género $(p=0,039)$, existiendo un mayor riesgo de desarrollar SBO en el género femenino RRR 5,32 (IC 95\% 1,10-25,66). En la subescala de despersonalización no hay diferencias estadísticamente significativas en 
Tabla 2. Variables estudiadas y presencia de síndrome de Burnout

\begin{tabular}{|c|c|c|c|c|c|c|}
\hline \multirow[t]{2}{*}{ Variable } & & \multirow[t]{2}{*}{ Total, n (\%) } & \multicolumn{4}{|c|}{ Presencia de Burnout, n (\%) } \\
\hline & & & Sí & & No & Valor p \\
\hline Edad & $\begin{array}{l}\leq 27 \text { años } \\
28-32 \text { años } \\
\geq 33 \text { años }\end{array}$ & $\begin{array}{l}30(29 \%) \\
50(49 \%) \\
23(22 \%)\end{array}$ & $\begin{array}{ll}25 & (83 \%) \\
35 & (70 \%) \\
15 & (65 \%)\end{array}$ & $\begin{array}{r}5 \\
15 \\
8\end{array}$ & $\begin{array}{l}(17 \%) \\
(30 \%) \\
(35 \%)\end{array}$ & 0,280 \\
\hline Género & $\begin{array}{l}\text { Masculino } \\
\text { Femenino } \\
\text { LGBT* }\end{array}$ & $\begin{array}{r}69(67 \%) \\
31(30 \%) \\
3 \quad(3 \%)\end{array}$ & $\begin{aligned} 48 & (69 \%) \\
25 & (80 \%) \\
2 & (66 \%)\end{aligned}$ & $\begin{array}{r}21 \\
6 \\
1\end{array}$ & $\begin{array}{l}(31 \%) \\
(20 \%) \\
(33 \%)\end{array}$ & 0,5 \\
\hline Estado civil & $\begin{array}{l}\text { Soltero } \\
\text { "Conviviente" } \\
\text { Casado } \\
\text { Separado }\end{array}$ & $\begin{array}{r}57(55 \%) \\
23(22 \%) \\
21(20 \%) \\
2(2 \%)\end{array}$ & $\begin{array}{r}42(73 \%) \\
19(82 \%) \\
12(60 \%) \\
2(100 \%)\end{array}$ & $\begin{array}{r}15 \\
4 \\
9 \\
0\end{array}$ & $\begin{array}{r}(27 \%) \\
(18 \%) \\
(40 \%) \\
(0 \%)\end{array}$ & 0,213 \\
\hline Año residencia & $\begin{array}{l}1^{\circ} \\
2^{\circ} \\
3^{\circ} \\
4^{\circ}\end{array}$ & $\begin{array}{r}25(24 \%) \\
35(34 \%) \\
41(40 \%) \\
2 \quad(2 \%)\end{array}$ & $\begin{aligned} 19 & (76 \%) \\
28 & (80 \%) \\
27 & (65 \%) \\
1 & (50 \%)\end{aligned}$ & $\begin{array}{r}6 \\
7 \\
14 \\
1\end{array}$ & $\begin{array}{l}(24 \%) \\
(20 \%) \\
(35 \%) \\
(59 \%)\end{array}$ & 0,463 \\
\hline Hijos & $\begin{array}{l}\text { No } \\
\text { Sí }\end{array}$ & $\begin{array}{l}81(78 \%) \\
22(22 \%)\end{array}$ & $\begin{array}{ll}57 & (70 \%) \\
18 & (82 \%)\end{array}$ & & $\begin{array}{l}(30 \%) \\
(18 \%)\end{array}$ & 0,285 \\
\hline Financiamiento** & $\begin{array}{l}\text { CONE-APS } \\
\text { CONESS } \\
\text { CONISS } \\
\text { EDF } \\
\text { FF.AA. } \\
\text { Universitario } \\
\text { Autofinanciado }\end{array}$ & $\begin{aligned} 5 & (4,8 \%) \\
7 & (6,7 \%) \\
34 & (33 \%) \\
15 & (14 \%) \\
2 & (2 \%) \\
9 & (8,7 \%) \\
23 & (22 \%)\end{aligned}$ & $\begin{array}{rr}5 & (100 \%) \\
6 & (85 \%) \\
24 & (70 \%) \\
9 & (60 \%) \\
0 & (0 \%) \\
6 & (66 \%) \\
19 & (82 \%)\end{array}$ & $\begin{array}{r}0 \\
1 \\
10 \\
6 \\
2 \\
3 \\
4\end{array}$ & $\begin{array}{l}(0 \%) \\
(15 \%) \\
(30 \%) \\
(40 \%) \\
(100 \%) \\
(33 \%) \\
(18 \%)\end{array}$ & 0,213 \\
\hline Región & $\begin{array}{l}\mathrm{RM}^{* * *} \\
\text { Otras regiones }\end{array}$ & $\begin{array}{l}79(76 \%) \\
24(23 \%)\end{array}$ & $\begin{array}{ll}59 & (75 \%) \\
16 & (66 \%)\end{array}$ & $\begin{array}{r}20 \\
8\end{array}$ & $\begin{array}{l}(25 \%) \\
(33 \%)\end{array}$ & 0,439 \\
\hline
\end{tabular}

* LGBT = lesbiana, gay, bisexual, transexual. ** Otros $=8$, 4 Servicio de salud, 1 institucional, 1 FORDIR y 2 no especifican. $* * * \mathrm{RM}=$ Región Metropolitana.

Tabla 3. Presencia de síndrome de Burnout según centro formador

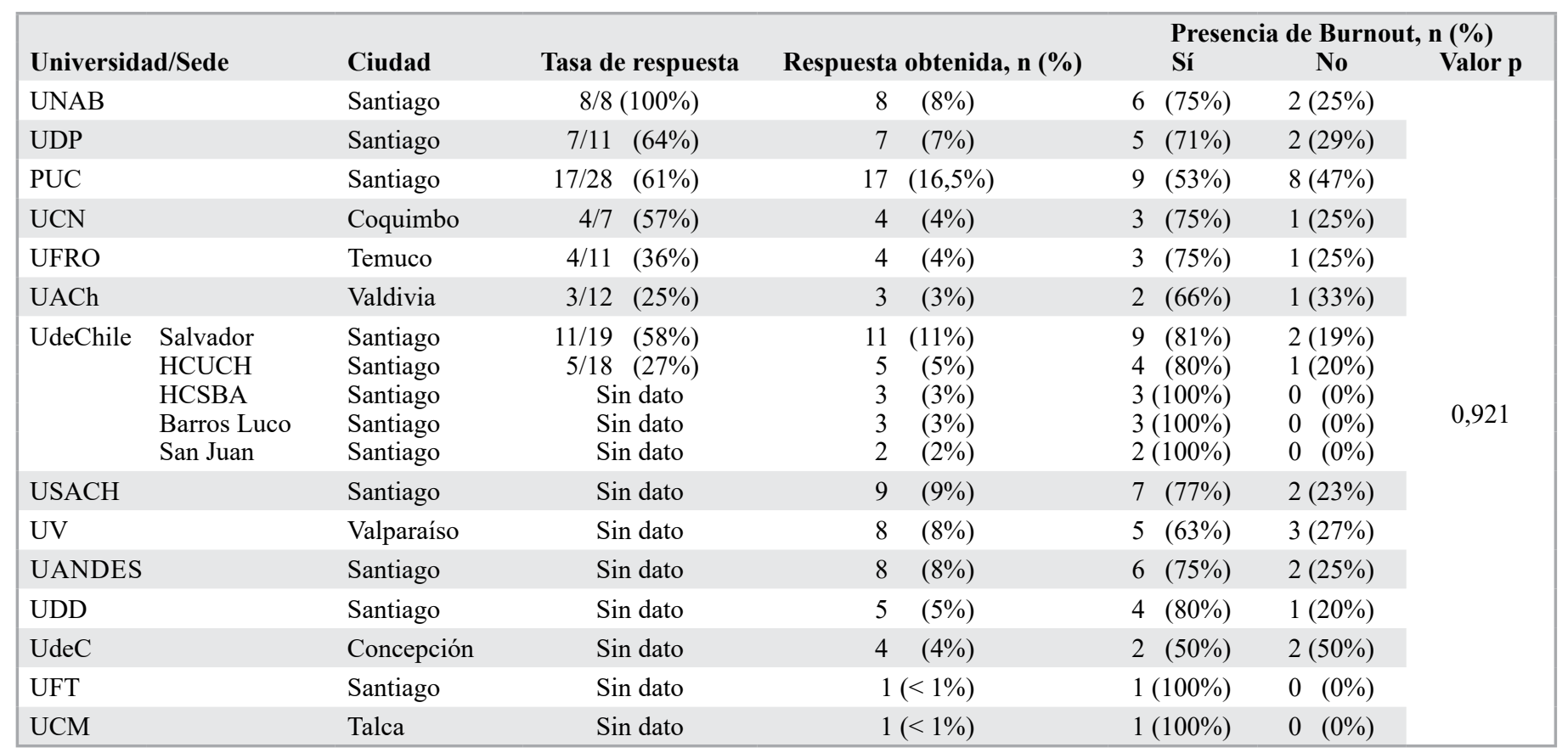


Tabla 4. Presencia de síndrome de Burnout en subescalas de Maslach y grado de éste

\begin{tabular}{|lccc|}
\hline & Agotamiento emocional & Despersonalización & Realización personal \\
\hline Alto n (\%) & $54(52 \%)$ & $58(56 \%)$ & $44(43 \%)$ \\
Moderado n (\%) & $30(29 \%)$ & $26(25 \%)$ & $28(27 \%)$ \\
Bajo n (\%) & $19(18 \%)$ & $19(18 \%)$ & $31(30 \%)$ \\
\hline
\end{tabular}

Tabla 5. Modelo de regresión logística multivariable de factores asociados a síndrome de Burnout

\begin{tabular}{|lcccc|}
\hline Variable & OR & Std. Err. & Valor $\mathbf{p}$ & IC 95\% \\
Edad & 0,802 & 0,867 & 0,042 & $0,64-0,99$ \\
Género & 0,527 & 0,305 & 0,270 & $0,16-1,64$ \\
Estado Civil & 0,859 & 0,456 & 0,775 & $0,30-2,43$ \\
Presencia hijos & 4,940 & 3,933 & 0,045 & $1,03-23,52$ \\
Santiago - Región & 1,714 & 0,959 & 0,335 & $0,57-5,13$ \\
Año Residencia & 0,975 & 0,324 & 0,940 & $0,50-1,87$ \\
Financiamiento & 1,237 & 0,456 & 0,543 & $0,84-2,45$ \\
Horas dedicación semanales & 0,554 & 0,253 & 0,196 & $0,22-1,35$ \\
\hline
\end{tabular}

Tabla 6. Análisis por subescalas y factores asociados a presencia de síndrome de Burnout (sólo se muestran aquellos factores con significancia estadística)

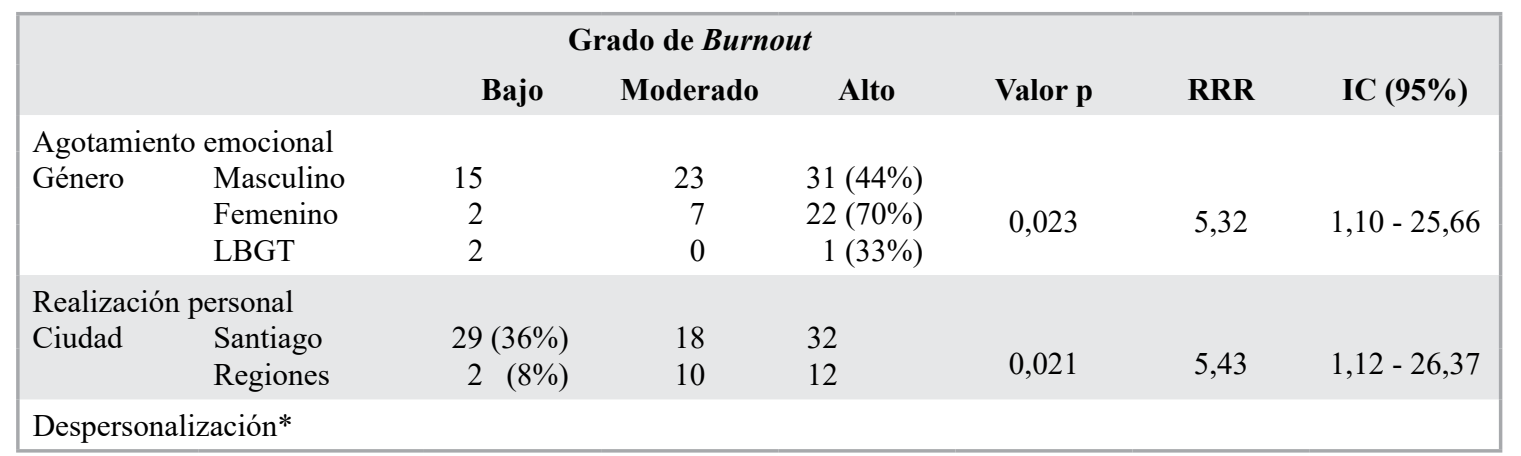

*Sin diferencias estadísticamente significativas.

las variables analizadas y en la subescala de realización personal existe una diferencia estadísticamente significativa al evaluar ciudad donde se realiza la formación ( $\mathrm{p}=0,021)$, existiendo un mayor riesgo de desarrollar SBO en quienes realizan la especialidad en Región Metropolitana RRR 5,43 (IC 95\% 1,12-26,37), con una baja realización personal.

\section{Discusión}

El SBO es más frecuente en personas que desempeñan trabajos con alta carga laboral y responsabili- dades, a quienes se les exige alto rendimiento y son sometidos a evaluaciones constantes. Estas características combinadas con algunos rasgos de personalidad, como tendencias perfeccionistas, excesiva tendencia al control con dificultad para delegar y alta competitividad, aumentan la probabilidad de sufrir SBO. Todas estas características mencionadas son comunes en residentes de especialidades médicas.

De las tres dimensiones que conforman el SBO, la más afectada fue la despersonalización (56\%), seguida del agotamiento emocional (52\%) y baja realización personal con un $(30 \%)$, estos datos son similares a otros trabajos reportados en literatura la- 
tinoamericana ${ }^{4,15}$ y que reflejan que las dos primeras son las que presentan mayor repercusión ${ }^{16}$.

Identificamos a la edad como un factor protector (OR 0,8 IC 95\% 0,649-0,991) de desarrollar SBO, la cual a su vez se correlaciona con el año de residencia. A mayor edad y año de residencia, menor SBO. Esta relación inversa se relaciona al desarrollo de madurez personal y profesional adquirida durante la formación.

La presencia de hijos se relacionó como factor de riesgo a la presencia de SBO (OR 4,9 IC 95\% 1,037$23,520)$, esto es discordante con otras series en que asocian la presencia de hijos como soporte fuera del ambiente hospitalario ${ }^{17}$ y que incluso aquellos sin hijos tienen 1,5 veces más SBO que aquellos con hijos ${ }^{18}$.

Llama la atención que el $80 \%$ de las mujeres presenten SBO en cualquier subescala (Tabla 1), independiente de no tener una diferencia significativa al compararlo con masculino y/o LBGT. Es una cifra preocupante, aunque concordante con la literatura internacional ${ }^{10}$. Este hallazgo se ve también en la dimensión de agotamiento emocional en que el $70 \%$ del género femenino presenta grado alto $v \mathrm{~s}$ el 44\% del género masculino ( $p=0,023$, RRR 5,32, IC 95\% 1,10-25,66). Esta mayor vulnerabilidad, ya se ha revelado en otros estudios y se explicaría por el hecho de que al incorporarse al mercado laboral, ellas sufren de doble jornada debido a sus roles familiares, del hogar y social ${ }^{4}$, esto sumado a que las residentes quirúrgicas femeninas son más propensas que las residentes no quirúrgicas a percibir un sesgo estereotipado contra las mujeres ${ }^{19}$.

No encontramos diferencias al evaluar el estado civil, así como tampoco al agruparlos en solteros y separados en un grupo, y convivientes y casados en otro, esto se contrasta con lo señalado por otras literaturas en que consideran la soltería como factor de riesgo de desarrollar $\mathrm{SBO}^{20}$, por el hecho de que los casados cuentan con una red de apoyo, que permitiría enfrentar mejor el estrés. Tampoco encontramos diferencias al evaluar el tipo de financiamiento ni tampoco al dicotomizarlos en con y sin financiamiento, ni tampoco cuanto al número de horas semanales dedicadas a la formación, sin embargo, nos llama la atención que el $40 \%$ declare dedicar más de 80 horas semanales a su residencia de cirugía general.

Es importante destacar que dentro de los diagnósticos diferenciales de la presencia de SBO, se debe considerar la depresión ${ }^{21}$ y que incluso la presencia de SBO se ha identificado como factor de riesgo para desarrollarla en médicos residentes ${ }^{22}$, llegando sus síntomas muchas veces a superponerse ${ }^{23}$.
Dentro de las fortalezas de nuestra investigación, queremos destacar que hasta el momento no existían datos oficiales relacionados con la prevalencia del SBO en residentes de cirugía de Chile. Consideramos que el realizar el esfuerzo de poder medirlo es el primer paso para luego prevenirlo y tratarlo. Dentro de las debilidades, está el hecho de no otorgar un resultado personal a los encuestados al finalizar el estudio, así como también el que nos hubiese gustado poder contar con una muestra de mayor tamaño, no obstante, se superó el número de encuestados calculados en el tamaño muestral. Precisamente el poder llegar con la encuesta a los residentes fue una de las dificultades que tuvimos. Hubo sedes (Tabla 3 ) en que lamentablemente el número de respuestas fue muy bajo y probablemente no es representativo de su realidad local.

Fuera de los objetivos formales del trabajo, creemos que se pueden realizar dos tipos de intervenciones para disminuir esta problemática, por una parte, centradas en el individuo y por otra, relacionada a cambios estratégicos organizacionales o estructurales. En este último punto vale la pena destacar el reconocimiento de la existencia del Burnout y su impacto en la atención de los pacientes, uso del conocimiento de los factores asociados al Burnout (objetivo secundario del presente estudio) e impulsar políticas de prevención, detección precoz y apoyo al estudiante con $\mathrm{SBO}$, lo que repercutirá en una mejor calidad de vida del educando y una mejor calidad de atención a los pacientes.

\section{Conclusión}

La prevalencia del síndrome de Burnout en becados de cirugía general de Chile es del 72,8\%.

En cuanto a los factores de riesgo, la edad es un factor protector; a medida que aumenta un año la edad, tienen 0,8 menos chances de tener SBO que los de menor edad. El tener hijos es un factor de riesgo importante; aquellos que tienen hijos tienen 4,94 veces más riesgo de tener SBO que aquellos que no los tienen.

En el análisis por subescalas, en la dimensión de agotamiento emocional se muestra que el género femenino, tiene 5,32 veces más riesgo de tener SBO que género masculino o LBGT. En la subescala de realización personal al analizar la ciudad donde se realiza la especialidad (Región Metropolitana $v s$ otras regiones), quienes la realizan en región metropolitana, tiene 5,43 veces más riesgo de desarrollar SBO que quienes la realizan en el resto del país. 


\section{Responsabilidades éticas}

Protección de personas y animales. Los autores declaran que para esta investigación no se han realizado experimentos en seres humanos ni en animales.

Confidencialidad de los datos. Los autores declaran que en este artículo no aparecen datos de pacientes.

Derecho a la privacidad y consentimiento in- formado. Los autores declaran que en este artículo no aparecen datos de pacientes.

Financiamiento: Sin apoyo financiero.

Conflictos de interés: No hay.

Agradecimientos: A cada uno de los residentes de cirugía general que respondieron esta encuesta.

\section{Referencias}

1. Maslach C, Schaufeli W, Leiter M. Job Burnout. Annu Rev Psychol. 2001;52:397422.

2. Leka S, Griffiths A, Cox T. La organización del trabajo y el estrés. Serie protección de la salud de los trabajadores $n^{\circ}$ 3. OMS. 2004;1-37.

3. Dewa C, Loong D, Bonato S, Thanh N, Jacobs P. How does burnout affect physician productivity? A systematic literature review. BMC Health Serv Res. 2014;14:325.

4. Terrones-Rodríguez J, Cisneros-Pérez V, Arreola-Rocha J. Burnout syndrome in medical residents at the General Hospital of Durango, México. Rev Med Inst Mex Seguro Soc. 2016;54:242-8.

5. Rodríguez A, Cruz M, Merino J. Burnout en profesionales de enfermería que trabajan en centros asistenciales de la octava región, Chile. Cienc y Enferm. 2008;14:75-85.

6. Barría J. Síndrome de Burnout en Asistentes Sociales del Servicio Nacional de Menores de la Región Metropolitana. PSYKHE. 2003;12:213-26.

7. Muñoz M, Molina P. Síndrome de Burnout en el Equipo de Salud de una Unidad de Cuidados Intensivos, Concepción. Rev Chil Med Intensiva 2013;28:13-8.
8. Romani M, Ashkar K. Burnout among physicians. Libyan J Med. 2014;9:1-6.

9. Rothenberger D. Physician Burnout and Well-Being. Dis Colon Rectum 2017;60:567-76.

10. Elmore L, Jeffe D, Jin L, Awad M, Turnbull I. National Survey of Burnout among US General Surgery Residents. J Am Coll Surg. 2016;223:440-51.

11. Cuestionario de Maslach Burnout Inventory [Internet]. Gabinete Psicologico- Javier Miravalles. [citado 5 de abril de 2018]. Disponible en: http:// www.javiermiravalles.es/sindrome burnout/Cuestionario de Maslach Burnout Inventory.pdf

12. Gil-Monte P. Validez factorial de la adaptación al español del Maslach Burnout Inventory-General Survey. Salud Publica Mex. 2002;44:33-40.

13. Manso-Pinto J. Estructura factorial de Maslach Burnout Inventory -Versión Human Services Survey- en Chile. Interam J Psychol. 2006;40:115-8.

14. Olivares V, Mena L, Jélvez C, Macía F. Validez factorial del Maslach Burnout Inventory Human Services (MBI-HSS) en profesionales Chilenos. Univ Psychol. 2014;13:145-59.

15. Azevedo L, Tonini C, Marques R, Dias L, Mancio E. Estrés y Burnout entre residentes multiprofesionales. Rev Lat Am Enfermagem. 2012;20:1-8.
16. Hernández C, Dickinson M, Fernández $\mathrm{M}$. El síndrome de desgaste profesional Burnout en medicos mexicanos. Rev Fac Med UNAM 2008;51:11-4.

17. Viswesvaran C, Sánchez J, Fisher J. The Role of Social Support in the Process of Work Stress: A Meta-Analysis. J Vocat Behav. 1999;54:314-34.

18. Martini S, Arfken C, Churchill A, Balon R. Burnout comparison among residents in different medical specialties. Acad Psychiatry 2004;28:240-2.

19. Salles A, Mueller C, Cohen G. Exploring the Relationship between Stereotypical Beliefs and Residents' Well-Being. J Am Coll Surg. 2016;8:583-92.

20. Hernández T, Terán $\mathrm{O}$, Navarrete $D$, León, A. El síndrome de Burnout: una aproximación hacia su conceptualización, antecedentes, modelos explicativos y de medición. Rev electrónica ICEA 2007;5:50-68.

21. Kaschka WP, Korczak D, Broich K. Burnout: a fashionable diagnosis. Dtsch Arztebl Int. 2011;108:781-7.

22. Pérez E. Síndrome de Burnout como factor de riesgo de depresión en médicos residentes. Med Int Mex. 2006;22:282-6

23. Wurm W, Vogel K, Holl A, Ebner C, Bayer D, Mörkl S, et al. DepressionBurnout Overlap in Physicians. PLoS One. 2016;11:1-15. 